American Journal of Applied Sciences 9 (7): 1091-1100, 2012

ISSN 1546-9239

(C) 2012 Science Publications

\title{
Properties of Chitosan Nanoparticles Formed Using Sulfate Anions as Crosslinking Bridges
}

\author{
Mayyas M.A. Al-Remawi \\ Department of Pharmaceutics, \\ College of Pharmacy, Taif University, Saudi Arabia
}

\begin{abstract}
Problem statement: The preparation of new simple Chitosan nanoparticle delivery system is very important and could have many applications particularly in pharmaceutical field to be used as a drug delivery system. In this study, the production of robust Chitosan nanoparticles has been developed and characterized by chemical crosslinking using sulfate anions. Approach: Chitosan polymer is considered one of the best polymers used in the field of Nanomedicine due to its safety, biocompatibility, biodegradability and environment friendly. Therefore, the development of a new method for the production of Chitosan nanoparticles should be of great importance for the pharmaceutical industry applications. The method was found to produce Chitosan sulfate capable carrying drug molecules which could explain the importance of such technique. Results: The size of Chitosan sulfate nanoparticles was determined using different LMW Chitosan $\mathrm{HCl}$ and sodium sulfate were confirmed by Laser diffraction, DSC and FTIR spectroscopy and it was tested for its dissolution rate. Conclusion/Recommendations: LMW Chitosan sulfate nanoparticles were relatively stable in aqueous medium and exert a slow rate of dissolution when placed in acidic medium. The properties of the Chitosan sulfate nanoparticles are considered suitable carriers in Nanomedicine and drug delivery technology.
\end{abstract}

Key words: Chitosan sulfate, sulfate nanoparticles, chemical cross-linking, drug delivery, sodium sulfate, drug molecules, Chitosan nanoparticles, particle size, pharmaceutical industry

\section{INTRODUCTION}

The production of nanoparticles is considered an important subject in applied sciences. Nanoparticles could be formed using metallic or polymeric compounds. The metallic nanoparticles include the use of gold, silver, iron, zinc and many other metallic compounds. Metallic nanoparticles found their application in a broad range of scientific fields (Mirkin and Taton, 2000; Salata, 2004; Yang et al., 2006; Watanabe et al., 2006). The polymeric nanoparticles usually formed of organic polymers of either natural or synthetic origin. The produced polymeric nanoparticles found their application mainly in Nanomedicine and Nanobiotechnology. The unique properties of polymeric nanoparticles encouraged their use as carriers for the development of therapeutic drug delivery systems. One interesting polymer which has been extensively used in the medical field is called Chitosan. It is either fully or partially deacetylated chitin. It is a natural, biocompatible, biodegradable and bioadhesive polymer. Chitosan molecular weight and degree of deacetylation are subjects of modifications to obtain different physico-mechanical properties. Low
Molecular Weight (LMW) chitosans are gaining the attention in the nanotechnology field due to their ability in forming nanoparticles (Qandil et al., 2009; Obaidat et al., 2010). However, the produced Chitosan nanoparticles were fragile in their structure and thus not suitable to be used without modification as carriers for drug molecules. The presence of free amine groups as well as hydroxyl groups of chitosans enables different chemical modifications to produce a relatively more rigid and robust chitosan structure. Different techniques have been used to modify Chitosan properties. One interesting technique was through the use of chemical structure modifiers including glutaraldehyde, glyoxal and polyethylene glycol (Kulkarni et al., 2005; Kildeeva et al., 2009; Wang and Stegemann, 2011). Another technique was through chemical interaction of positively charged Chitosan with other negatively charged materials as for example in case of gelation with alginates or xanthan gum or sodium lauryl sulfate or oleic acid (Ali et al., 2005a; 2005b; Elsayed et al., 2011). However, these techniques shall either modify the chemical structure of Chitosan or produce a combination of two polymeric materials and this might raise many questions in the regulatory bodies regarding 
interactions and material safety. A simpler technique can be used through chitosan salt formation where some anions may cause chitosan crosslinking via ionic interactions. This would be a more convincing solution for the pharmaceutical industry. There are many examples of such crosslinkers including tripolyphosphate, sodium citrate, amino acids, sodium sulfate (Rana et al., 2005; Bhumkar and Pokharkar, 2006; Beppu et al., 2007; Tsai et al.,, 2007).

The use of sodium sulfate anions in the crosslinking of LMW Chitosan has been scarcely described in the literature. The use of sulfate anion for the development of Chitosan nanoparticles and thus the formulation of nanoparticles has not been fully investigated in the literature to the best of our knowledge.

In this study attempts are made to prepare and characterize LMW Chitosan nanoparticles formed using sulfate anions as crosslinking bridges.

\section{MATERIALS AND METHODS}

Chitosan was purchased from Xiamen Xing, China. Chitosan degree of deacetylation was $93 \%$. Chitosan base has an average molecular weight around $250 \mathrm{kDa}$. Analytical grade sodium sulfate (anhydrous) was used. Deionized water was used throughout the experiment.

Preparation of LMW Chitosan HCl: Three different LMW Chitosan $\mathrm{HCl}$ were prepared according to a previously published method (Qandil et al., 2009). The viscosity average molecular weight and the Degree of Deacetyl Ation (DDA) were determined based on viscosity method and ${ }^{1} \mathrm{H}$ NMR technique, respectively. The molecular weights of Chitosan that has been used in the study were 6,13 and $30 \mathrm{kDa}$. All Chitosan grades were found to be completely deacetylated i.e., $100 \%$ DDA (Lavertu et al., 2003).

Preparation of chitosan sulfate nanoparticles: The crosslinking formation was prepared by the ionic interaction between the positively charged functional groups of chitosan and the sulfate anions of sodium sulfate.

$1000 \mathrm{mg}$ chitosan $\mathrm{HCl}$, of the three molecular weight $(6,13$ and $30 \mathrm{kDa}$, were dissolved in separate Erlenmeyer flasks containing $100 \mathrm{~mL}$ of deionized water to produce chitosan $\mathrm{HCl}$ solutions. Another solution was obtained via dissolving $3600 \mathrm{mg}$ of sodium sulfate in $100 \mathrm{~mL}$ deionized water. The fixed portions of were withdrawn from sodium sulfate solutions and each portion was added gradually to a separate beaker containing $100 \mathrm{ml}$ of $1 \%$ Chitosan $\mathrm{HCl}$ solutions of each molecular weight and mixed vigorously using magnetic stirring.

Optical microscope: Few drops of chitosan $\mathrm{HCl}$ solution $\left(80 \mathrm{mg} \mathrm{mL}^{-1}\right.$ ) were put on slides and either freeze dried or dried in oven at $75^{\circ} \mathrm{C}$ until complete dryness. Another few liquid drops of each crosslinked Chitosan sulfate particulates were dispersed in a solution of different molecular weight and at the same concentration were placed on microscopic glass slides and left to dry in a closed oven at $75^{\circ} \mathrm{C}$ prior to get a microscopic photo (Nikon LV150, with Camera, Japan) for the particles formed on the surface of the slide after drying.

Turbidimetric measurements: The turbidity was measured using a spectrophotometer (Beckman Du640 , Germany) at a fixed wavelength of $650 \mathrm{NM}$ with a $1-\mathrm{cm}$ path length quartz cuvette at $25^{\circ} \mathrm{C}$. The turbidity measurements were first calibrated against deionized water. All samples were mixed with magnetic stirring prior to measurements. After mixing, the transmittance corresponding had been always stable with respect to turbidity measurements. The transmittance of each chitosan $\mathrm{HCl}$ sample after the addition of sodium sulfate was measured and converted into turbidity (100-\%T). The blank reading for each sample was measured for the corresponding chitosan $\mathrm{HCl}$ and an added portion of $\mathrm{NaCl}$ as expected from the addition reaction. Similarly, Glucosamine $\mathrm{HCl}$ solution was prepared and considered as a reference material (Miura et al., 1999).

Particle size measurements: Samples of chitosan $\mathrm{HCl}$ before the addition of sodium sulfate and after the addition of sodium sulfate at the same chitosan (based on glucosamine units) to sodium sulfate molar ratios of 2: 1 and 4: 1 were assessed by Malvern Zetasizer NanoZS series (Malvern Instruments, UK). The average and standard deviations were calculated and the viscosity was determined using a Vibro Viscometer (SV-10, A\&D Company, Japan).

Infra-red Spectra: The crosslinked chitosans prepared by 2:1 moL ratio of chitosan having monomeric glucosamine $\mathrm{HCl}$ to sodium sulfate of different molecular weights were dried and used for FTIR analysis. Other Chitosan $\mathrm{HCl}$ samples were prepared with the same corresponding amount of $\mathrm{NaCl}$ dried in oven at $105^{\circ} \mathrm{C}$ for $3 \mathrm{~h}$ prior to testing via Infra-red spectroscopy. The Infra Red (IR) spectra was tested via FTIR (Perkin-Elmer, UK). Five milligrams of the analyst were geometrically mixed with about $100 \mathrm{mg}$ of dry $\mathrm{KBr}$ and appropriate amounts of and disks were prepared by compression. All samples were scanned in the wave number region from 4000 and $400 \mathrm{~cm}^{-1}$. 
Thermal studies: About $5 \mathrm{mg}$ of each chitosan sulfate powder as prepared previously was placed in differential scanning calorimetry (DSC, Mettler Toledo DSC 820, Germany). Similarly, the corresponding Chitosan $\mathrm{HCl}$ samples were also scanned by DSC for comparison purposes. The sample to be analyzed using DSC aluminum pan. The samples were heated starting from room temperature up to $300^{\circ} \mathrm{C}$ at a rate of $10^{\circ} \mathrm{C} \mathrm{min}$. The DSC apparatus was calibrated using Indium metal.

Nanoparticle dissolution study: Crosslinked Chitosan sulfate particulate dispersed in solution with molecular weight $6 \mathrm{kDa}$ was monitored for its change in particle size during dissolution in $0.1 \mathrm{M} \mathrm{HCl}$ and in another neutralized medium achieved through neutralizing 0.1 $\mathrm{M} \mathrm{HCl}$ with $0.1 \mathrm{M} \mathrm{NaOH}$ to reach a $\mathrm{pH}$ of neutrality i.e., 7. Buffering agents such as phosphate buffer has been excluded to avoid any possibility of interaction with Chitosan. The crosslinked chitosans prepared by 2 : 1 mol ratio of chitosan having monomeric glucosamine $\mathrm{HCl}$ to sodium sulfate of molecular weight $6 \mathrm{kDa}$ present as a liquid dispersion. Other Chitosan $\mathrm{HCl}$ sample of the same molecular weight was prepared with the same corresponding amount of $\mathrm{NaCl}$. Dissolution procedure includes the addition of $20 \mathrm{~mL}$ crosslinked chitosan liquid dispersion to $80 \mathrm{~mL}$ of $0.1 \mathrm{M} \mathrm{HCl}$. The samples were put in $250 \mathrm{~mL}$ Erlenmeyer flask, a water bath shaker at $37^{\circ} \mathrm{C}$ at 50 strokes $\min ^{-1}$ was used. Samples were withdrawn from the flask every 15minute and the particle size was measured using Malvern Nanosizer after equilibrated to a temperature of $25^{\circ} \mathrm{C}$. The same procedure was repeated at the neutralized medium. Another chitosan $\mathrm{HCl}$ sample containing the corresponding percent of $\mathrm{NaCl}$ was also used for comparison purposes.

Statistical treatment: For group comparison, A Single-Way Analysis of Variance (ANOVA) was carried out. When the probability value $(\mathrm{P})$ was less than 0.05 the difference was considered statistically significant.

\section{RESULTS}

LMW Chitosan was prepared by acid hydrolysis according to previously published study (Qandil et al., 2009). Sodium sulfate was added to Chitosan $\mathrm{HCl}$ in order to make crosslinked Chitosan sulfate derivative. Physical properties such as swellability was demonstrated between chitosan $\mathrm{HCl}$ and crosslinked chitosan sulfate. The salt samples were wetted with small amount of water then mixed with large amounts of water, Fig. 1. The LMW Chitosan $\mathrm{HCl}$ liquid was dried using freeze drier or in oven at $75^{\circ} \mathrm{C}$ and microscopic photos were taken for each molecular weight, Fig. 2.
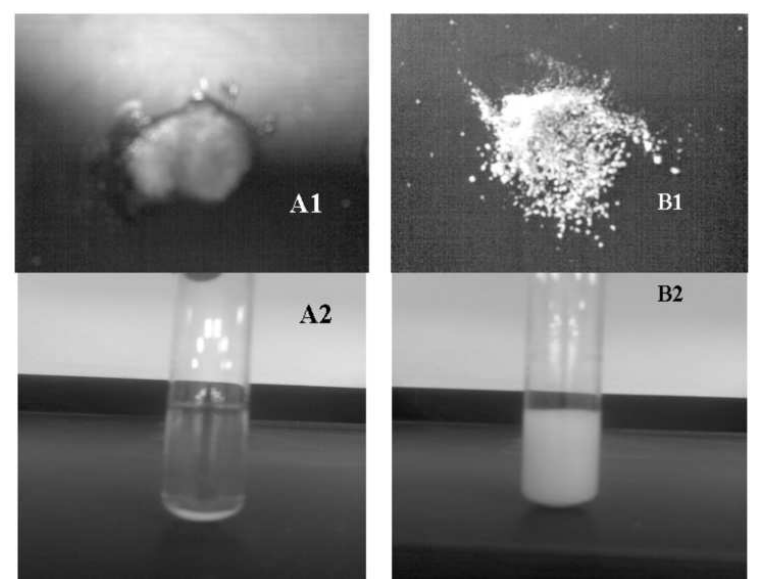

Fig. 1: Photographs taken for Chitosan $\mathrm{HCl}$ (A) and Chitosan sulfate (B) upon wetting with a minute amount of water and after the addition of large amounts of water

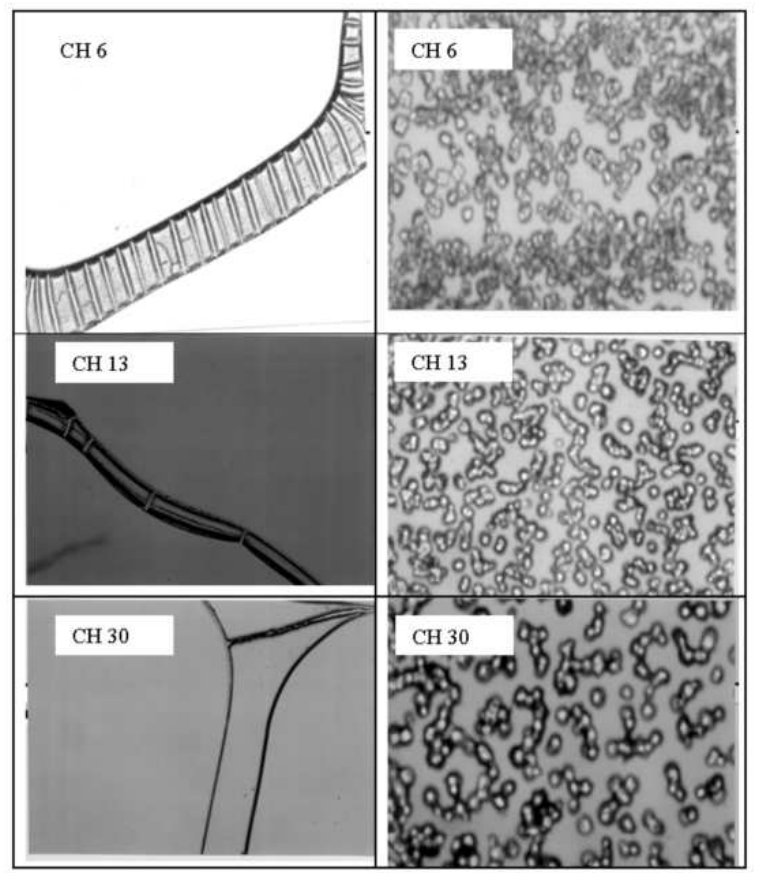

Fig. 2: Microscopic photos ((magnification 200x)) for chitosan $\mathrm{HCl}$ 6, 13 and $30 \mathrm{kDa}$ dissolved in water. The samples were then freeze dried (left side column) and dried in oven at $75^{\circ} \mathrm{C}$ in (the right side column) 


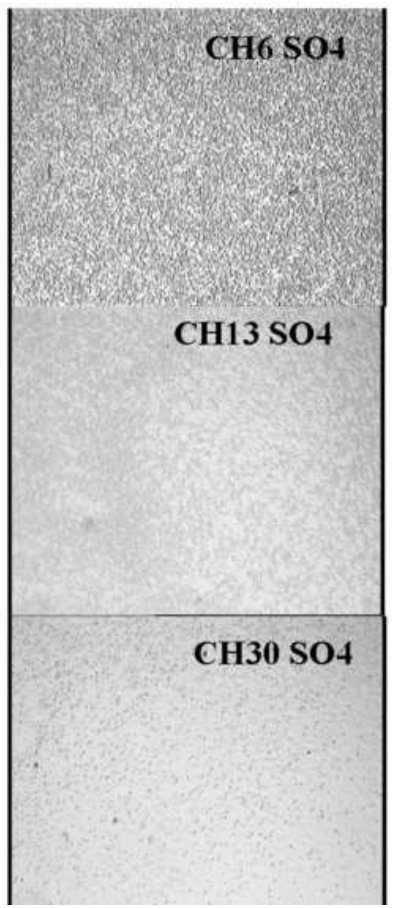

Fig. 3: Optical microscopic photographs (magnification 40x) of LMW crosslinked Chitosan sulfate 6,13 and 30 dried in oven at $75^{\circ} \mathrm{C}$

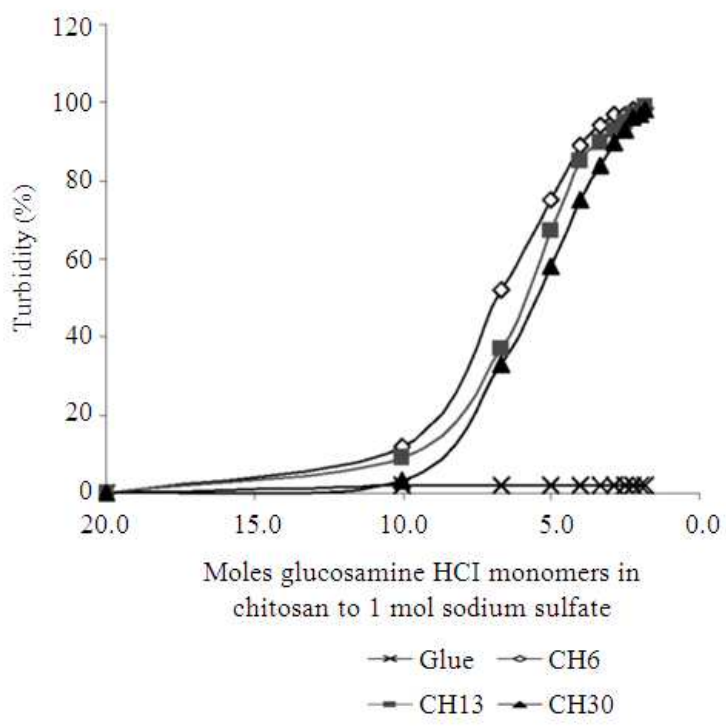

Fig. 4: Turbidity of LMW chitosans and glucosamine $\mathrm{HCl}$ upon the addition of sodium sulfate

The crosslinked Chitosan sulfate aggregated particles were evaluated under optical microscope of different molecular weight Chitosan as shown in Fig. 3.

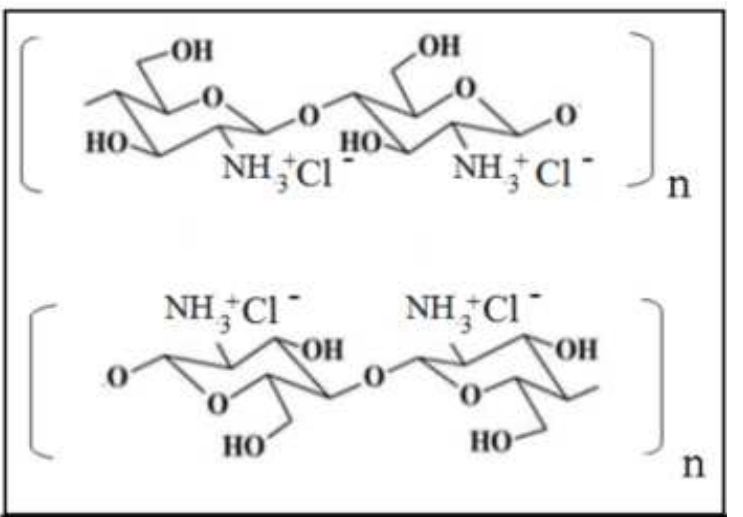

(A)

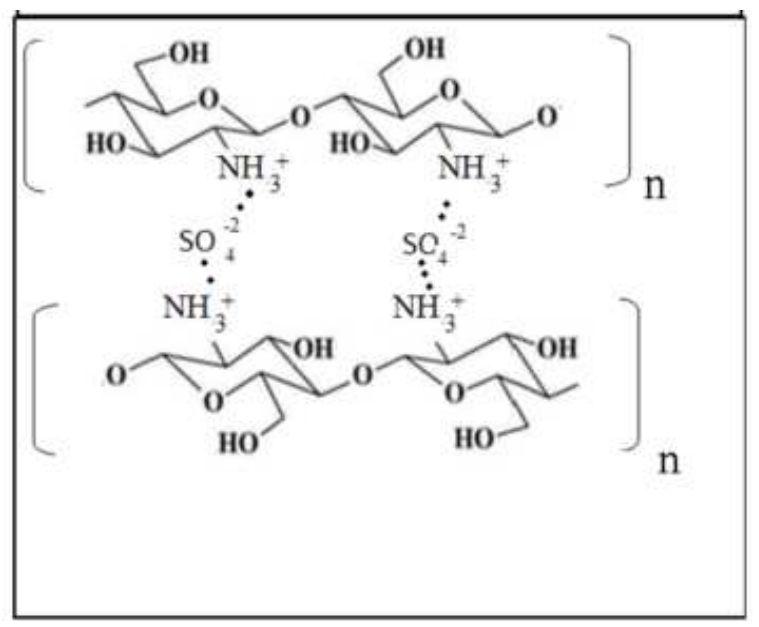

(B)

Fig. 5: Chemical structure of chitosan (polyglucosamine) $\mathrm{HCl}$ (A) and presentation of crosslinking reaction between positively charged amine groups of chitosan (polyglucosamine) $\mathrm{HCl}$ and sulfate anions (B)

The formation of chitosan sulfate nanoparticles were observed simply by the increase in the turbidity in aqueous solution of chitosan $\mathrm{HCl}$ upon the addition of sodium sulfate as shown in Fig. 4. A schematic presentation of the crosslinking reaction between chitosan primary amine functional groups and sulfate anions is shown in Fig. 5. Changes in particle size of Chitosan $\mathrm{HCl}$ before and after addition of sodium sulfate were determined as shown in Fig. 6. The crosslinking reaction was confirmed by FTIR and DSC as shown in Fig. 7 and 8, respectively. In vitro dissolution study was conducted to demonstrate stability against simulated gastric and intestinal fluids as depicted in Fig. 9. 


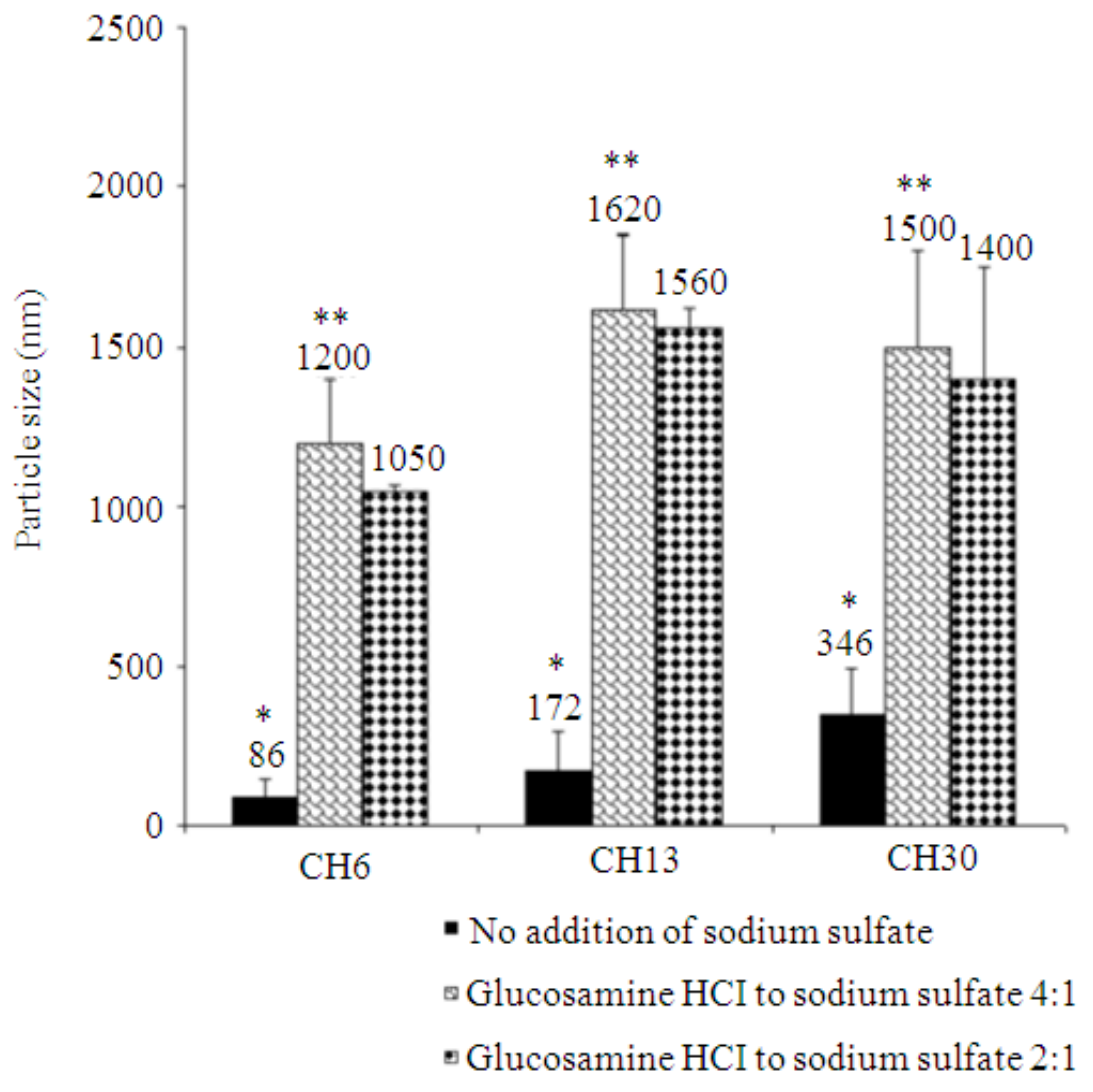

Fig. 6: Particle size of Chitosan upon the addition of sodium sulfate at molar ratios (based on Chitosan monomer weight) and sodium sulfate $2: 1$ and $4: 1, \mathrm{P}$ value $<0.05$

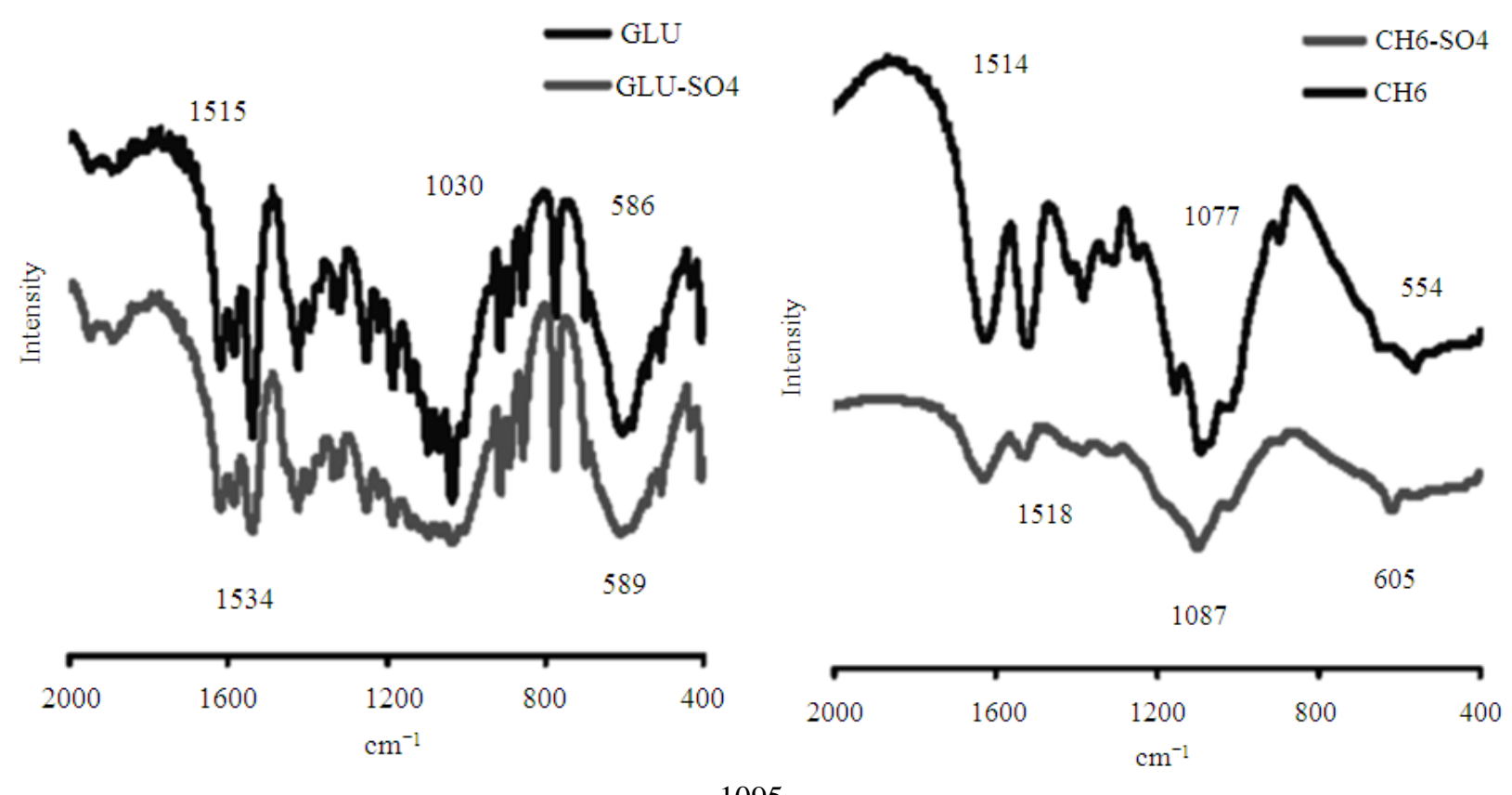


Am. J. Applied Sci., 9 (7): 1091-1100, 2012
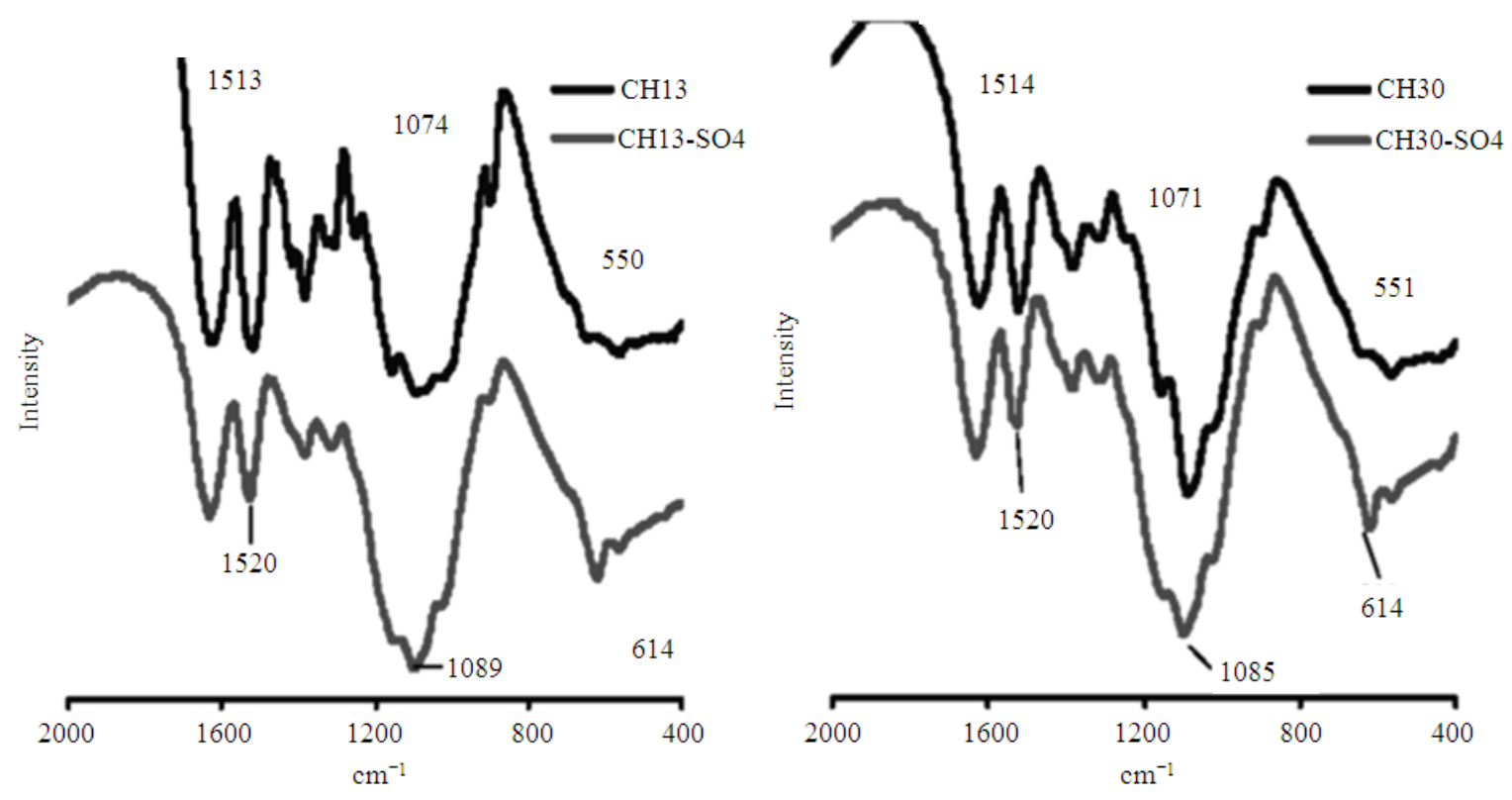

Fig. 7: FTIR of glucosamine and LMW chitosans in form of hydrochloride and sulfate salts

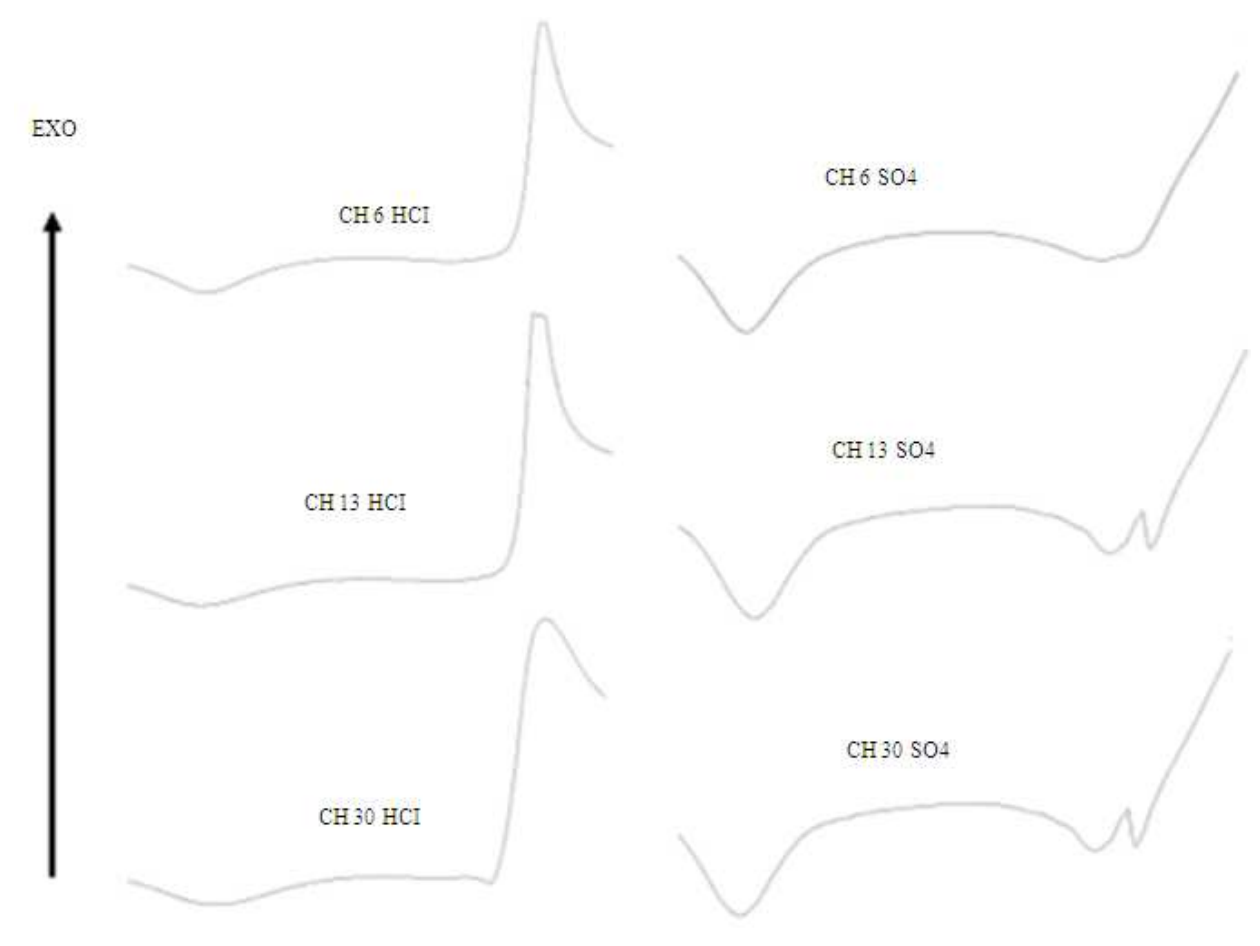

$406080100120140160180200220240260280 \quad 4060 \quad 80100120140160180200220240260280$

Fig. 8: DSC thermograms of LMW chitosans in form of hydrochloride and sulfate salts 
Am. J. Applied Sci., 9 (7): 1091-1100, 2012

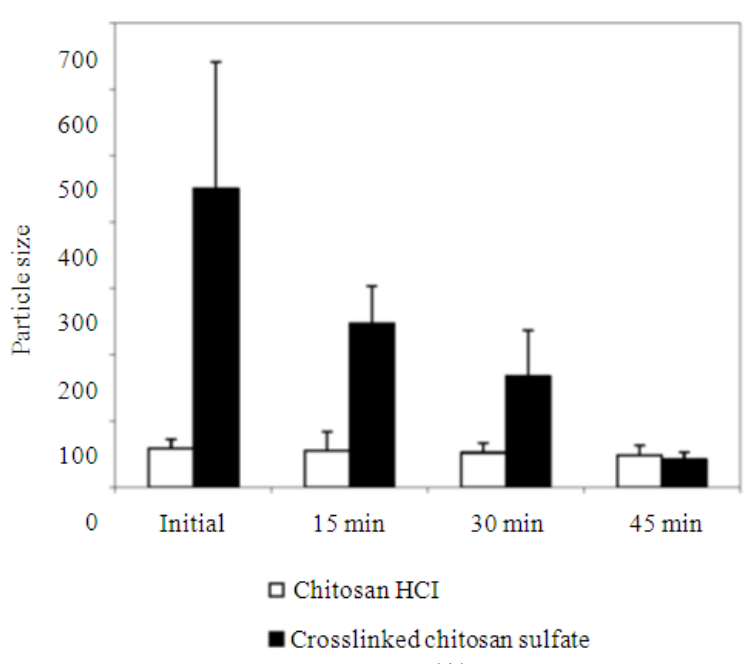

(A)

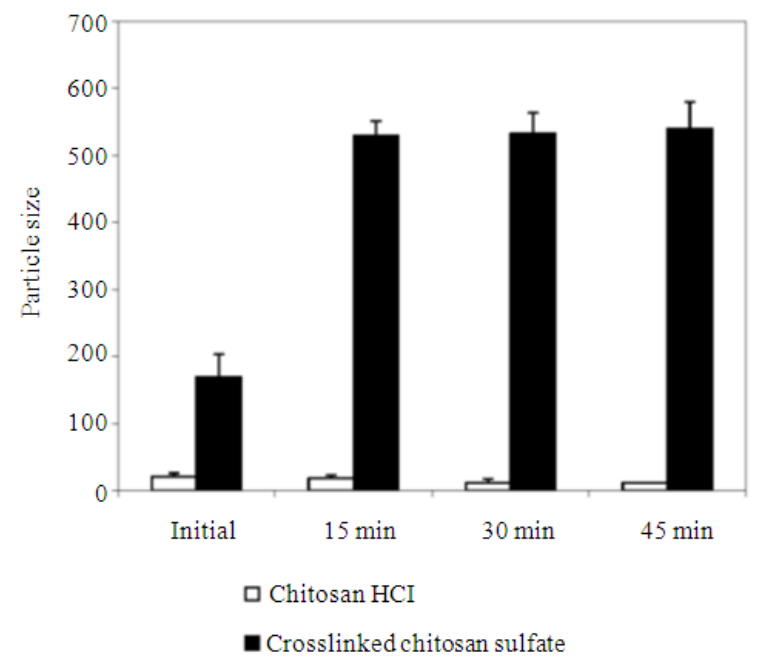

(B)

Fig. 9: Dissolution of crosslinked chitosan $6 \mathrm{kDa}$ with sodium sulfate particles in $0.1 \mathrm{M} \mathrm{HCl}$ and after neutralization (pH 7) medium

\section{DISCUSSION}

On a macroscopic level, Chitosan $\mathrm{HCl}$ upon wetting with a minute amount of water it resulted in a swollen material which was dissolved with large quantity of water and produced a transparent solution with very small particulates. However, the crosslinked Chitosan sulfate exhibited the formation of aggregated masses which produced a turbid liquid upon the addition of a large quantity of water, Fig 1. At the microscopic level, LMW Chitosan $\mathrm{HCl}$ of different molecular weight forms different structural forms in the solution as shown in Fig. 2. The method of drying is very important to get into a clear picture about Chitosan conformation or type of aggregation in solution state. It seems that freezing Chitosan solution then drying at very low temperature gives a more realistic picture about Chitosan conformation in the aqueous solution. Accordingly, Chitosan $\mathrm{HCl}$ forms cylindrical aggregates and the size of such aggregates was dependent on the molecular weight of Chitosan. As of Chitosan $\mathrm{HCl}$ molecular weight decreases, the formed aggregates become smaller and denser or more compacted. In case of drying of Chitosan in oven at higher temperature, the resulted aggregates of Chitosan were smaller and their size becomes oval-like particles. This finding agrees well with previous study by (Tsaih and Chen, 1997), where they discovered that Chitosan conformations depends on molecular weight. When molecular weights are high i.e., Above $223 \mathrm{kDa}$, Chitosan will acquire a random coil shape. While, when chitosan molecular weights are low i.e., below $148 \mathrm{kDa}$, chitosan will form a rod shape structure. These changes in conformation were attributed to the changes in hydrogen bonding and due to the difference in the charge distribution among high and LMW compounds. Similarly, when the Chitosan crosslinking reaction occurred via the addition of sulfate the formed aggregated particulates were found to be dependent on Chitosan molecular weight where Chitosan sulfate with the lowest molecular weight i.e., $6 \mathrm{kDa}$ showed the highest number and most dense aggregates as shown in Fig. 3. This could be due to the aggregation of Chitosan sulfate into particles that would do the other steps of aggregation upon drying to form micronized particles. In addition, chitosan sulfate $6 \mathrm{kDa}$ looks to be darker than the other molecular weights under the microscope field when investigated under the same conditions i.e., The light intensity of the microscope did not change, the amount of Chitosan added to the solution was the same and the drying process was also the same. The experiment has been repeated several times and the results were the same.

The addition of sodium sulfate to Chitosan $\mathrm{HCl}$ solution resulted in the appearance of turbidity, which was dependent on the amount of sodium sulfate present in the solution, Fig. 4. This would be explained based on the formation of crosslinking reaction. The negatively charged sulfate divalent anions would form electrostatic interactions with the positively charged amine functional groups of the present glucosamine units in two chitosan strands, Fig. 5. In case of free glucosamine $\mathrm{HCl}$, sodium sulfate and even the formed glucosamine sulfate all of these products are known to 
be water-soluble and so there could be almost no significant increase in the turbidity or even a weak electrolyte formation might be produced. Thus, the simple electrostatic interaction is not the main determining factor for turbidity but the formation of cross-linked Chitosan which produces water-insoluble particles possibly due to the presence of Glucosamine units in a chain-like structures. This opens the door for the chain length of Chitosan to play an important role in this type of aggregation. Different LMW chitosans showed different degrees of turbidity upon the addition of sodium sulfate. Where, CH6 $\mathrm{kDa}$ produced the highest degree of turbidity, while $\mathrm{CH} 30$ produced the lowest degree of turbidity. These results agree well with the previous photos in microscopic observations. It is known that Chitosan is a straight chain Glucosamine $\mathrm{HCl}$ polymer which is present in water as nanoparticles of either extended rod-like structure or as a spherical structure depending on concentration, $\mathrm{pH}$, Ionic strength, molecular weight (Obaidat et al., 2010). Due to the high degree of solubility of LMW Chitosan $\mathrm{HCl}$, they possess very little turbidity in water. The crosslinking of LMW Chitosan $\mathrm{HCl}$ with sulfate anions could decrease their solubility and produce more compacted particles with less flexibility of the chains. This enhances the formation of compacted nanoparticles systems. In conclusion, the results indicated the importance of the presence of Glucosamine in polymeric structure. In addition, the number of repeating Glucosamine units could have different degree of aggregation pattern during the crosslinking reaction. Turbidity increases with the continuous addition of sodium sulfate, where maximum turbidity of solution occurred after the addition of a molar ratio of about 4 or 2 moles of Chitosan (polyglucosamine) $\mathrm{HCl}$ to 1 mole sodium sulfate. Consequently, the particle size of this solution was determined on these ratios, Fig. 6. LMW chitosans are present in agglomerated nanosized particles before the addition of sodium sulfate and their particle size was found to be dependent on their molecular weight. The addition of sodium sulfate resulted in an increase in the particle size of the produced Chitosan sulfate. The particle size did not change significantly between the two monomer molar ratios of Chitosan to sodium sulfate $4: 1$ and 2:1. It was found that the degree of increase in particle size from Chitosan $\mathrm{HCl}$ to Chitosan sulfate was about 14-4 fold for $\mathrm{CH} 6, \mathrm{CH} 13$ and $\mathrm{CH} 30$ under the same conditions, respectively. This means that further aggregation of the particles could occur in case of lower molecular weight chitosans. The aggregation of lower sized particles into larger particles could result in smaller sized inter-particle spaces, which could result in a more restriction for the light passage. This would explain the increase in turbidity upon crosslinking of smallest molecular weight chitosan.

Since the produced LMW chitosan is 100\% deacetylated it does not contain amide functional groups which are usually found in high molecular weight chitosan. Thus, the behavior of LMW chitosan would be much similar to the primary amine structures. It is known that the primary amine functional group shows $\mathrm{N}-\mathrm{H}$ stretching at 3500-3200 and also $\mathrm{N}-\mathrm{H}$ bend at $1550-1450 \mathrm{~nm}$, Fig. 7. However, due to water molecules interference, the region 4000-2000 was excluded from the FTIR spectrum. On the other hand for sodium sulfate it is known that FTIR of sodium sulfate has symmetric stretching for $\mathrm{SO}_{4}$ at $1123 \mathrm{~nm}$ and asymmetric bending of $\mathrm{SO}_{4}$ at $615 \mathrm{~nm}$ (Periasamy et al., 2009). In case of chitosan sulfate one can observe the presence of the two peaks that could correspond to the presence of sulfate in the regions of $615 \mathrm{~nm}$ and $1100 \mathrm{~nm}$. However, the shift of the $1513 \mathrm{~nm}$ of the amino group bend into higher wave number may indicate a higher energy level for all crosslinked LMW chitosan sulfates. This would suggest the chemical interaction with sulfate anion which could restrict the bending of the $\mathrm{N}-\mathrm{H}$ group. It was reported that hydrogen bonding is known to lead to shifting of the N$\mathrm{H}$ deformation band to higher frequencies. As this shift was also described for chitosan sulfate, it may be related to the establishment of ionic bonding between the cationic primary amine groups and sulfate anions. Consequently, this may suggest the presence of crosslinking reaction between sulfate anions and ammonium groups of chitosan (Granja et al., 2004).

In DSC studies, all Chitosan $\mathrm{HCl}$ samples showed decomposition before melting while all Chitosan sulfate samples were melted prior to decomposition, Fig. 8. This is in agreement with the ammonium chloride material which has a very high melting point i.e. above $500^{\circ} \mathrm{C}$, while ammonium sulfate has a much lower melting point $235-280^{\circ} \mathrm{C}$. Consequently, Chitosan ammonium sulfate functional groups may exert melting prior to decomposition of the polymer (Jariwala et al., 2007).

In conclusion, $\mathrm{CH} 6 \mathrm{kDa}$ would be more compact when compared with chitosan 13 and $30 \mathrm{kDa}$. Thus, chitosan $6 \mathrm{kDa}$ would be more preferable to be used as a carrier for drug transportation. The main goal for the nanoparticles of Chitosan sulfate is to play as drug carriers when administered to humans. Chitosan sulfate as a biocompatible material can be administered to humans safely through many routes of administration however; the most important route would be the oral route. In oral route, the particles will be exposed to 
acidic conditions of the stomach and then to the neutral conditions of the small intestine. An in vitro dissolution study can be used to simulate the changes in $\mathrm{pH}$ where $\mathrm{CH} 6$ code has been selected because it was the most firm or united form among all used Chitosan molecular weights as shown in Fig. 9. Acidic conditions were found to dissolve the nanoparticles of Chitosan sulfate and convert it to lower particle size. However, it was found that more than 30 minutes were required in order to complete the conversion. The gastric transit time of nanoparticles present in liquid formulation will be very short time. However, the bioadhesion and penetration enhancement properties of Chitosan may play important role in drug delivery (Davis, 2005). This may suggest an improvement in drug transport when Chitosan sulfate is used as an oral carrier system. Once the particles reach the small intestine the $\mathrm{pH}$ will be changed to neutral, in this $\mathrm{pH}$ range the particles will not dissolve easily on the contrary a further aggregation was observed for Chitosan sulfate. It was reported that Chitosan nanoparticles may take a randomly coiled conformation at alkaline/neutral $\mathrm{pH}$, because of their unionized amino groups (Berger et al., 2004), while at lower $\mathrm{pH}$ region, Chitosan acquires a net positive charge due to protonation of amino groups and swelling can occur as a result of electrostatic repulsion of the charged ions group. Similar findings were also observed upon dispersing Chitosan sodium lauryl sulfate nanoparticles in neutral $\mathrm{pH}$ medium (Elsayed $e t$ al., 2009).

\section{CONCLUSION}

LMW chitosans can form aggregated structures in aqueous solution using sulfate anions as crosslinking agent. These aggregated particles can be used as drug carriers. However, it is of high importance to take into consideration Chitosan molecular weight effect on the physical properties of the formed particles.

\section{ACKNOWLEDGEMENT}

Dr. Adnan Badwan (Jordanian Pharmaceutical Manufacturing company) is highly acknowledged for his continuous support.

\section{REFERENCES}

Ali, B.A., A.R.M.M. Ahma and S.M.B.A.W. Sheikh, 2005a. Universal controlled-release composition comprising chitosan-EPO Patent EP1512394. IPEXL.
Ali, B.A., A.R.M.M. Ahma and S.M.B.A.W. Sheikh, 2005b. Universal controlled-release composition comprising xanthan gum and sodium alginate EPO Patent EP1510205. IPEXL.

Beppu, M.M., R.S. Vieira, C.G. Aimoli and C.C. Santan, 2007. Crosslinking of chitosan membranes using glutaraldehyde: Effect on ion permeability and water absorption. J. Membr. Sci., 301: 126130. DOI: 10.1016/j.memsci.2007.06.015

Berger, J., M. Reist, J.M. Mayer, O. Felt and R. Gurny, 2004. Structure and interactions in Chitosan hydrogels formed by complexation or aggregation for biomedical applications. Eur. J. Pharm. Biopharm., 57: 35-52. DOI: 10.1016/S09396411(03)00160-7

Bhumkar, D.R. and V.B. Pokharkar, 2006. Studies on effect of $\mathrm{pH}$ on cross-linking of Chitosan with sodium tripolyphosphate: A technical note. AAPS Pharm. Sci. Tech., 7: E50-E50. PMID: 16796367

Davis, S.S., 2005. Formulation strategies for absorption windows. Drug Dis. Today, 10: 249-257. DOI: 10.1016/S1359-6446(04)03351-3

Elsayed, A., M. Al-Remawi, N. Qinna, A. Farouk and A. Badwan, 2009. Formulation and characterization of an oily-based system for oral delivery of insulin. Eur. J. Pharm. Biopharm., 73: 269-279. DOI: 10.1016/j.ejpb.2009.06.004

Elsayed, A., M. Al-Remawi, N. Qinna, A. Farouk and K.A.ejpbSou'od et al., 2011. Chitosan-sodium lauryl sulfate nanoparticles as a carrier system for the in vivo delivery of oral insulin. AAPS Pharm., 12: 958-964. DOI: 10.1208/s12249-011-9647-5

Granja, P.L., A.I.N. Silva, J.P. Borges, C.C. Barrias and I.F. Amaral, 2004. Preparation and characterization of injectable Chitosan-hydroxyapatite microspheres. Key Eng. Mat., 254-256: 573-576. DOI: $\quad$ 10.4028/www.scientific.net/KEM.254256.573

Jariwala, M., J. Crawford and D.J. LeCaptain, 2007. In situ Raman spectroscopic analysis of the regeneration of ammonium hydrogen sulfate from ammonium sulfate. Indu. Eng. Chem. Res., 46: 4900-4905. DOI: 10.1021/ie070350v

Kildeeva, N.R., P.A. Perminov, L.V. Vladimirov, V.V. Novikov and S.N. Mikhailov, 2009. About mechanism of Chitosan cross-linking with glutaraldehyde. Russ. J. Bioorg. Chem., 35: 360369. DOI: $10.1134 / \mathrm{S} 106816200903011 \mathrm{X}$

Kulkarni, A.R., V.I. Hukkeri, H.W. Sung and H.F. Liang, 2005. A novel method for the synthesis of the PEG-crosslinked Chitosan with a pHindependent swelling behavior. Macromol. Biosci., 5: 925-928. DOI: 10.1002/Mabi.200500048 
Lavertu, M., Z. Xia, A.N. Serreqi, M. Berrada and A. Rodrigues et al., 2003. A validated ${ }^{1} \mathrm{H}$ NMR method for the determination of the degree of deacetylation of Chitosan. J. Pharm. Biomed. Anal., 32: 1149-1158. DOI: 10.1016/S07317085(03)00155-9

Mirkin, C.A. and T.A. Taton, 2000. Materials chemistry: Semiconductors meet biology. Nature, 405: 626-627.

Miura, N., P.L. Dubin, C.N. Moorefield and G.R. Newkome, 1999. Complex formation by electrostatic interaction between carboxylterminated dendrimers and oppositely charged polyelectrolytes. Langmuir, 15: 4245-4250. DOI: 10.1021/la9901251

Obaidat, R., N. Al-Jbour, K. Al-Sou'd, K. Sweidan and M. Al-Remawi et al., 2010. Some physicochemical properties of low molecular weight chitosans and their relationship to conformation in aqueous solution. J. Solut. Chem., 39: 575-588. DOI: $10.1007 / \mathrm{s} 10953-010-9517-\mathrm{x}$

Periasamy, A., S. Muruganand and M. Palaniswamy, 2009. Vibrational studies of $\mathrm{Na}_{2} \mathrm{SO}_{4}, \mathrm{~K}_{2} \mathrm{SO}_{4}$, $\mathrm{NaHSO}_{4}$ and $\mathrm{KHSO}_{4}$ crystals. Rasayan J. Chem., 2: 981-989.

Qandil, A.M., A.A. Obaidat, M.A.M. Ali, B.M. AlTaani and B.M. Tashtoush et al., 2009. Investigation of the interactions in complexes of low molecular weight Chitosan with Ibuprofen. J. Solut. Chem., 38: 695-712. DOI: 10.1007/s10953009-9405-4

Rana, V., K. Babita, D. Goyal and A. Tiwary, 2005. Sodium citrate cross-linked Chitosan films: optimization as substitute for human/rat/rabbit epidermal sheets. J. Pharm. Pharmaceut. Sci., 8: 10-17.
Salata, O.V., 2004. Nanoparticles-known and unknown health risks. J. Nanobiotechnol., 2: 1-12. DOI: 10.1186/1477-3155-2-12

Tsai, S.P., C.Y. Hsieh, C.Y. Hsieh, D.M. Wang and L.L.H. Huang et al., 2007. Preparation and cell compatibility evaluation of Chitosan/collagen composite scaffolds using amino acids as crosslinking bridges. J. Applied Polym. Sci., 105: 1774-1785. DOI: 10.1002/app.26157

Tsaih, M.L. and R.H.. Chen, 1997. Effect of molecular weight and urea on the conformation of Chitosan molecules in dilute solutions. Int. J. Biol. Macromol., 20: 233-240. DOI: 10.1016/S01418130(97)01165-3

Wang, L. and J.P. Stegemann, 2011. Glyoxal crosslinking of cell-seeded chitosan/collagen hydrogels for bone regeneration. Acta Biomater., 7: 2410-2417. DOI: 10.1016/j.actbio.2011.02.029

Watanabe, K., D. Menzel, N. Nilius and H.J. Freund, 2006. Photochemistry on metal nanoparticles. ChemInform. DOI: 10.1002/chin.200652229

Yang, P., W. Zhang, Y. Du and X. Wang, 2006. Hydrogenation of nitrobenzenes catalyzed by platinum nanoparticle core-polyaryl ether trisacetic acid ammonium chloride dendrimer shell nanocomposite. J. Mol. Catal. A: Chemical, 260: 410. DOI: 10.1016/j.molcata.2006.06.045 